\title{
Weight Minimization of Helical Compression Spring Using Gravitational Search Algorithm (GSA)
}

\author{
Amer Noor Hafizah", a , Ahmad Nurhidayati ${ }^{1, b}$ and Zainal Abidin Amar Faiz ${ }^{2, c}$ \\ ${ }^{1}$ Department of Mechanical Engineering, Faculty of Engineering, National Defense University of \\ Malaysia, Sungai Besi Army Camp, 57000 Kuala Lumpur, Malaysia \\ ${ }^{2}$ Faculty of Electrical Engineering, Universiti Teknologi MARA, 81750 Pasir Gudang, Johor, \\ MALAYSIA \\ a, ${ }^{*}$ noorhafizah@upnm.edu.my, ${ }^{\mathrm{b}}$ nurhidayati7491@gmail.com, ${ }^{\mathrm{c}}$ amarf408@johor.uitm.edu.my
}

\begin{abstract}
Keywords: Compression Spring, Weight minimization, Gravitational Search Algorithm, Constrained Optimization
\end{abstract}

\begin{abstract}
Compression spring is one of the most common mechanical componet being used in most mechanisms. Many criteria and constraints should be considered in designing and specifying the spring dimensions. Therefore, it has been one of the standard case studies considered to test a new optimisation algorithm. This paper introduced an optimization method named Gravitational search Algorithm (GSA) to solve the problem of weight minimization of spring. From previous studies, weight minimization of a spring has been investigated by many researcher using various optimization algorithm technique. The result of this study were compared to one of the previous studies using Particle Swarm Optimization (PSO) algorithm. Also, parametric studies were conducted to select the best values of GSA parameters, beta and epsilon. From the results obtained, it was observed that the optimum dimensions and weight obtained by GSA are better than the values obtained by PSO. The best values of beta and epsilon was found to be 0.6 and 0.01 respectively.
\end{abstract}

\section{Introduction}

Spring is an elastic device that is used to store mechanical potential energy. A mechanical spring used to exert force, provide flexibility and to store and absorb energy. The suspension spring give high frequency fluctuating load and impulsive load during the driving process in automobile which operates as a buffering and shock absorber. So, a good quality of suspension spring plays important role in the stationary and safety in automobile. Therefore, helical compression spring with a good flexibility and can absorb great energy is widely used in automotive industry where the overall weight plays an important role to the overall efficiency of the vehicle. Therefore, minimizing the weight is usually the main objective in designing any automotive component.

A process of finding maximum or minimum for function which depends on some parameters was defined as design optimization. The optimization is carried out when there are some designs variables need to be considered to obtain maximum or minimum parameters and it must also satisfy some constraints. Several approach for an optimization was introduced such as analytical or numerical method and advanced optimization technique such as Genetic Algorithm (GA) [1], Particle Swarm Optimization (PSO), Ant Colony Algorithm (ACA), Artificial Bee Colony (ABC) Algorithm and Evolutionary Algorithm [2]. All these methods use mathematical programming such as MATLAB software techniques to carry out the algorithm for the optimization solutions.

Hence, optimal design of spring is solved by using Gravitational Search Algorithm. GSA is basically derived from the law of gravity. GSA is found to have a better result compared to PSO [3] because it has a potential in solving a discrete engineering optimization problem. The objective of this project is to minimize the weight of spring design by using Gravitational Search Algorithm and its performance.

Next section will discuss the characteristics of spring design and the concept of GSA application in solving engineering problems. 


\section{Spring Design}

Different type of spring possess different properties and function indeed but the main function still the same where mechanical springs are used to exert force, to store or absorb energy, and to provide flexibility. The optimal design which is to minimize the weight itself cannot be analyzed directly due to the variety of types and functions of the springs. Therefore, this study has been set to focus on a small scaled helical compression spring which is commonly used in automotive industrial such as for a suspension spring. This spring are useful in the operation of many devices due to several desirable properties, such as a near linear rate and also the availability of the materials that can be used to fabricate them, and the ease of manufacture. For this reason helical compression springs have been in used for some time.

A spring are widely used in industrial because the differences of the properties and functions. In 2010, John L. Porteiro in his paper of optimization of spring design with fatigue proposed a fundamental of spring design [4]. According to this paper, it considers only helical compression spring because it is the most common used for suspension in automotive fields. This type of spring is very useful for many devices due to desirable properties such as near linear rate (particularly after the first $20 \%$ of deflection). In his paper, a design variable is considered for an optimal design for a spring.

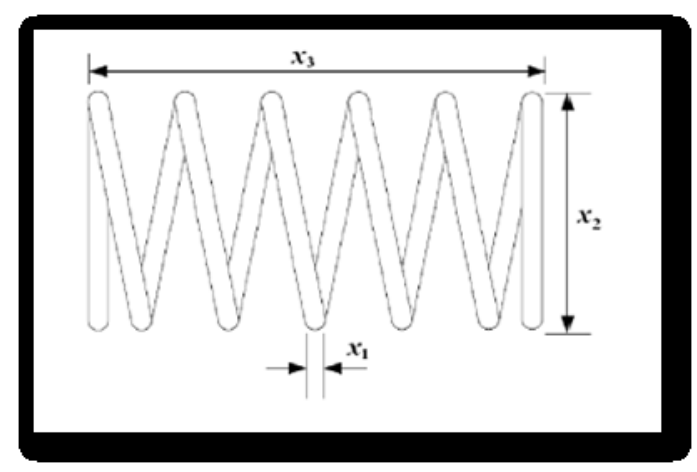

Fig. 1Tension/compression spring [3]

\section{Gravitational Search Algorithm}

In 2009, Esmat Rashedi [5] developed a new optimization algorithm named Gravitational Search Algorithm (GSA). The idea based on the Newton's law of gravity and law of motion where all the objects will attract each other by its own gravitational force. This gravitational force causes the objects to move toward the objects with heavier masses. Then, masses will cooperate using a direct form of communication, through gravitational force. The heavier masses correspond to good solution and move slower. Light masses correspond to poor solution and move faster toward heavy masses. In GSA, there are four parameters for each agent (mass): position, inertial mass, active gravitational mass and passive gravitational mass. By using the Newton's law of motion, the velocity of each agent is updated after the acceleration is calculated.

Similar with PSO, some of the position and velocity updating methods for each agent are employed here. For each iteration, the position of each agent (mass) is updated using the modified velocity. This position will give an updated solution for the optimization problem through a fitness function represented by the agent's gravitational and inertial masses.

Assume position of each agent in the searching space is represented by $X_{i}=\left(x_{i}^{1}, x_{i}^{2}, x_{i}^{3} \ldots, x_{i}^{n}\right)$ for $i=1,2,3, \ldots, N i=1,2,3, \ldots, N$. Here, $N$ represents the number of agents and the position of $i^{\text {th }}$ agents in the $d^{\text {th }}$ dimension can be represented as $x_{i}^{4}$.

Using the equality of gravitational and inertia mass assumption, $\left(M_{a i}=M_{p i}=M_{i i}=M_{i}\right)$ the mass value, $M$ is then calculated for updating each $i^{\text {th }}$ agent using Eq (1) : 


$$
M_{i}(t)=\frac{m_{i}(t)}{\sum_{j=1}^{N} m_{j}(t)}
$$

Here, $m$ can be determined using Eq (2):

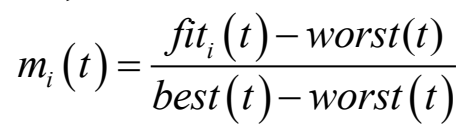

For a minimization problem, the definition of best (t) and worst ( $t$ ) are defined as in Eq (3):

$$
\begin{aligned}
& \operatorname{best}(t)=\min _{j \in\{1, \ldots . ., N\}} f i t_{j}(t) \\
& \operatorname{worst}(t)=\max _{j \in\{1, \ldots \ldots, N\}} f i t_{j}(t)
\end{aligned}
$$

The fitness value, $f_{i t}(t)$ affects the mass value of $i$ th agents, which corresponds to the position of the particle in a search space. Next, the fitness, $f i t{ }_{j}(t)$, is evaluated for each agent according to the objective function as later explained in Eq (8). The gravitational constant, $G(t)$, is updated due to the effect of decreasing gravity. The best and the worst values of the population were calculated.

$$
G(t)=G\left(t_{0}\right) \times\left(\frac{t_{0}}{t}\right)^{\beta} \quad, \beta<1
$$

Mass, $M$ for each agent is calculated and the acceleration, $\alpha$ was calculated using $\alpha_{i}^{d}(t)=F_{i}^{d}(t)$ and the force acting is calculated as follows:

$$
\begin{aligned}
& F_{i}^{d}(t)=\sum_{j=1, j \neq i}^{N} \operatorname{rand}_{j} F_{i j}{ }^{d}(t) \\
& F_{i j}^{d}(t)=G(t) \frac{M_{a j}(t)}{R_{i j}(t)+\varepsilon}\left(x_{j}^{d}(t)-x_{i}^{d}(t)\right)
\end{aligned}
$$

where $M_{a j}$ is the active gravitational mass related to agent $j, \varepsilon$ is small constant, $R_{i j}$ is the distance between agent $i$ and $j$, and rand ${ }_{j}$ is a uniform random variable in the interval $[0,1]$. Then the velocity, $v_{i}^{d}$ and position $x_{i}^{d}$, of $i$ th agents is calculated as follows:

$$
\begin{aligned}
& v_{d}^{i}(t+1)=\operatorname{rand}_{i} \times v_{d}^{i}(t)+\alpha_{i}^{d}(t) \\
& x_{d}^{i}(t+1)=x_{d}^{i}(t)+v_{d}^{i}(t+1)
\end{aligned}
$$

where, rand $_{i}$ is a uniform random number in the interval $[0,1]$. This updating process is repeated as long as the stopping criterion is not satisfied. The procedure can be summarized in Figure 2.

\section{Simulation Parameters}

This research is using simulation methods extensively. A simulation is carried out by using Gravitational Search Algorithm (GSA) to get the best optimal design of spring with the minimum weight. The algorithm optimizes the design variables for the helical compression spring model (wire diameter, the mean coil diameter, the number of active coils) that will affect the overall weight of the designed spring. These variables are optimized subject to certain constraints based on the mechanical properties of the spring. The optimum weight is then compared against the optimum weight by using PSO from previous study [3]. For a better perspective on the organization of this study, a flow chart of the study was constructed and can be shown in the Figure 3.

The benchmark fitness functions $(\mathrm{Eq}(8))$, design variables and constraints for the spring design are taken from [3]. The three design variables and the subsequent operable range are shown in Table 1. Initial setting parameters used for the simulation are shown in Table 2. 


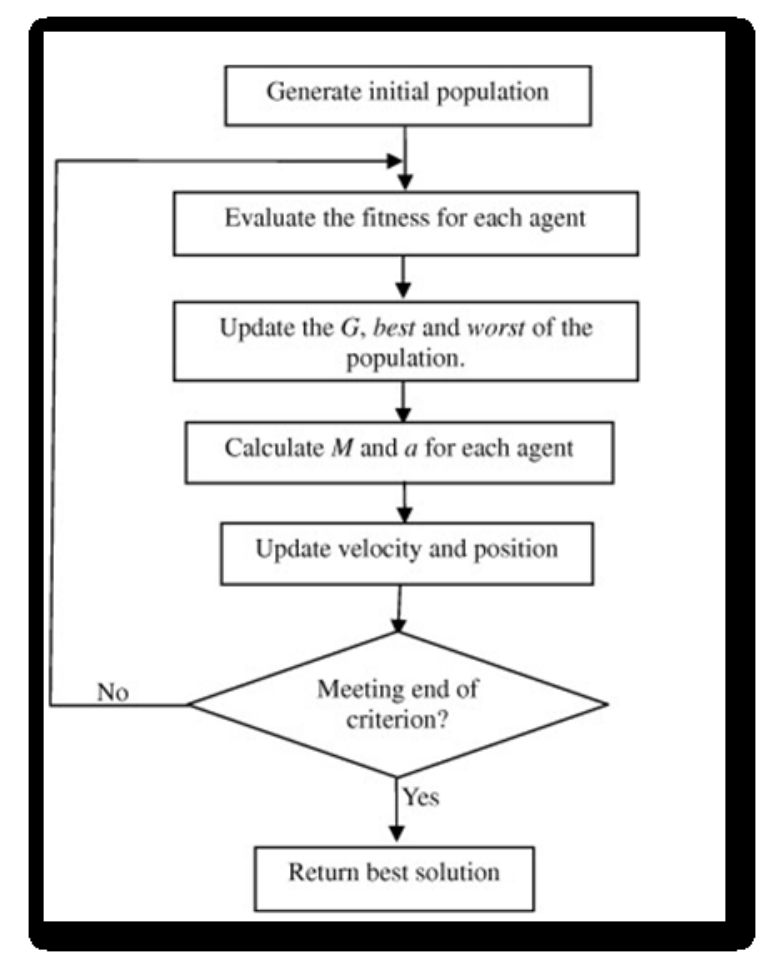

Minimize, $f(\bar{x})=\left(x_{3}+2\right) x_{2} x_{1}^{2}\left(\right.$ inch $\left.^{3}\right)$

Table 1 Spring design variables [3]

\begin{tabular}{|c|c|}
\hline Design variable & Range \\
\hline Wire diameter, $x_{1}$ (inch) & $0.05 \leq x_{1} \leq 2$ \\
\hline $\begin{array}{c}\text { Mean coil diameter, } x_{2} \\
\text { (inch) }\end{array}$ & $0.25 \leq x_{2} \leq 1.3$ \\
\hline Number of active coil, $x_{3}$ & $2 \leq x_{3} \leq 15$ \\
\hline
\end{tabular}

Fig. 2 Flowchart of the GSA [6]

These variables are subjected to four (4) design constraints as listed below:

Minimumdeflection, $g_{1}(\bar{x}): 1-\frac{x_{2}{ }^{3} x_{3}}{7178 x_{1}{ }^{4}} \leq 0$
Shear stress, $g_{2}(\bar{x}): \frac{4 x_{2}{ }^{2}-x_{1} x_{2}}{12566\left(x_{2} x_{1}{ }^{3}\right)-x_{1}{ }^{4}}+\frac{1}{5108 x_{1}^{2}}-1 \leq 0$
Surge frequency, $g_{3}(\bar{x}): 1-\frac{140.45 x_{1}}{x_{2}{ }^{2} x_{3}} \leq 0$
Limits on the outside diameter, $g_{4}(\bar{x}): \frac{x_{2}+x_{1}}{1.5}-1 \leq$
\begin{tabular}{|c|c|}
\hline \\
Table 2 Initial parameters for GSA simulation [3] \\
\hline Parameters & Values \\
\hline Number of agents, $N$ & 500 \\
\hline Number of iteration & 0.1 \\
\hline Bheta, $\beta$ & 0.01 \\
\hline Epsilon, $\varepsilon$ & 4 \\
\hline Number of constraints & 3 \\
\hline Number of dimension, $i$ &
\end{tabular}

\section{Results and Discussions}

Using the parameters and constraints defined before, GSA algorithm was carried out to optimize the weight of a helical spring. MATLAB programming environment was employed Table 3 shows the solution produced by the GSA for the initial run with initial parameters as tabulated in Table 2. These results were compared against previous solution using PSO by [3]. The results proven that GSA 
managed to obtain better value for the spring overall volume $\left(\mathrm{inch}^{3}\right)$ which directly corresponds to the overall weight. There was $53.85 \%$ decrease in the overall volume using the optimized dimension from GSA algorithm compared to the PSO solution by [3]. All variables were optimized and well kept within operable range while satisfying the four constraints before. The comparison and the best solution can be shown as tabulated in Table 3 below.

Table 3 Result for GSA optimization with initial parameters

\begin{tabular}{|c|c|c|c|}
\hline & PSO [3] & GSA & \% Difference \\
\hline $\boldsymbol{x}_{\boldsymbol{1}}$ (inch) & 0.051583 & 0.056442 & $9.42 \%$ \\
\hline $\boldsymbol{x}_{\boldsymbol{2}}$ (inch) & 0.35419 & 0.330414 & $-6.71 \%$ \\
\hline $\boldsymbol{x}_{\boldsymbol{3}}$ (inch) & 11.438675 & 3.55223 & $-68.95 \%$ \\
\hline $\boldsymbol{g}_{\boldsymbol{1}}$ & $-2.00 \mathrm{E}-16$ & -0.75899 & - \\
\hline $\boldsymbol{g}_{\boldsymbol{2}}$ & $-1.00 \mathrm{E}-16$ & -0.37858 & - \\
\hline $\boldsymbol{g}_{\boldsymbol{3}}$ & -4.048765 & -19.4412 & $380.18 \%$ \\
\hline $\boldsymbol{g}_{\boldsymbol{4}}$ & -0.729483 & -0.7421 & $1.73 \%$ \\
\hline $\boldsymbol{f ( x )}$ (inch $^{3}$ ) & 0.012665 & 0.005844 & $-53.85 \%$ \\
\hline
\end{tabular}

\section{Conclusion}

In optimizing a spring design, a method using GSA algorithm has been proposed in this study. Using the readily available parameters and constraints, the GSA algorithm has been employed and an optimized design has been obtained. The GSA method proposed have been validated against previous results [3] and evaluated. According to the overall result, GSA performed better result compared to PSO. 53.85\% of volume improvement has been recorded for the optimized spring design. This algorithm has a good prospect for future development. Further study on the effect of GSA parameters Beta and Epsilon can be carried out to determine the effect on the algorithm in terms of the quality and also stability of the solutions. Choosing the right values for these parameters is one of the most important aspects in employing this algorithm. This study has been adapting previous values used by previous studies. Also, the proposed GSA method can be implemented in solving high dimensional and complex optimization problems due to the fact that this algorithm can perform better than the more established PSO algorithm.

\section{References}

[1] M. Satyanarayana, et al. (November 2012). Optimization of Spring Weight Using Genetic Algorithm. International Journal of Engineering Research \& Technology (IJERT).

[2] Efren Mezura-Montes, Engineering Optimization Using a Simple Evolutionary Algorithm. mexico: Evolutionary Computation Group (EVOCINV).

[3] Cagnina, L. C., Esquivel, S. C., Nacional, U., Luis, D. S., Luis, S., \& Coello, C. A. C. (2008). Solving Engineering Optimization Problems with the Simple Constrained Particle Swarm Optimizer. SiC-PSO, 32, 319-326

[4] Porteiro, J. L. (2010). Spring design optimization with fatigue..

[5] Esmat Rashedi, H. N.-p. (2009). GSA: A Gravitational Search Algorithm. Information Sciences, 2232-2248

[6] A. Bhattacharya, S. Datta, and M. Basu, "Gravitational Search Algorithm Optimization for Short-term Hydrothermal Scheduling," in 2012 International Conference on Emerging Trends in Electrical Engineering and Energy Management (ICETEEEM), (2012), pp. 216-221 